\title{
An experimental investigation of mixing mechanisms in shock-accelerated flow
}

\author{
C. TOMKINS, S. KUMAR, G. ORLICZ AND K. PRESTRIDGE \\ Physics Division, Los Alamos National Laboratory, \\ Los Alamos, NM 87545, USA
}

(Received 27 November 2006 and in revised form 28 May 2008)

An experimental investigation of mixing mechanisms in a shock-induced instability flow is described. We obtain quantitative two-dimensional maps of the heavy-gas $\left(\mathrm{SF}_{6}\right)$ concentration using planar laser-induced fluorescence for the case of a shockaccelerated cylinder of heavy gas in air. The instantaneous scalar dissipation rate, or mixing rate, $\chi$, is estimated experimentally for the first time in this type of flow, and used to identify the regions of most intense post-shock mixing and examine the underlying mechanisms. We observe instability growth in certain regions of the flow beginning at intermediate times. The mixing rate results show that while these unstable regions play a significant role in the mixing process, a large amount of mixing also occurs by mechanisms directly associated with the primary instability, including gradient intensification via the large-scale strain field in a particular non-turbulent region of the flow.

\section{Introduction}

The Rayleigh-Taylor Instability (RTI) occurs across a perturbed interface between two fluids when a pressure gradient opposes the interfacial density gradient (Taylor 1950). An analogue to RTI, known as Richtmyer-Meshkov Instablity (RMI), occurs when a density interface is impulsively accelerated, e.g. by a shock wave (Richtmyer 1960; Meshkov 1969). In this case, there is a deposition of vorticity by the shock wave associated with the baroclinic term in the vorticity equation, $\nabla \rho \times \nabla p$, if the density gradient (associated with the interface) and the pressure gradient (from the shock) are misaligned. RMI appears in a wide range of problems, including the collapse of supernovae (Arnett et al. 1989), implosion of inertial confinement fusion capsules (Lindl, McCrory \& Campbell 1992), and supersonic combustion (Yang, Kubota \& Zukowski 1993).

In the classic single-interface RMI problem, a brief linear growth regime occurs while the amplitude of the disturbance is small relative to the characteristic wavelength. Non-linear growth follows, characterized by interpenetration of the two fluids with spikes and bubbles. Eventually, secondary instabilities develop, aiding the nonlinearities in breaking down the motions into progressively smaller scales and, if the initial input of energy is sufficient, transitioning the flow to turbulence. The largeand small-scale motions present in the flow field distort the interface and enhance the diffusive mixing of the two fluids. Reviews by Brouillette (2002) and Zabusky (1999) offer more comprehensive descriptions of the problem.

A shock-accelerated gaseous cylinder is a simplified test problem that allows the study of certain aspects of RMI. (See figure 1 for a schematic of the experimental configuration, discussed in detail in the following section.) Although the linear theory 


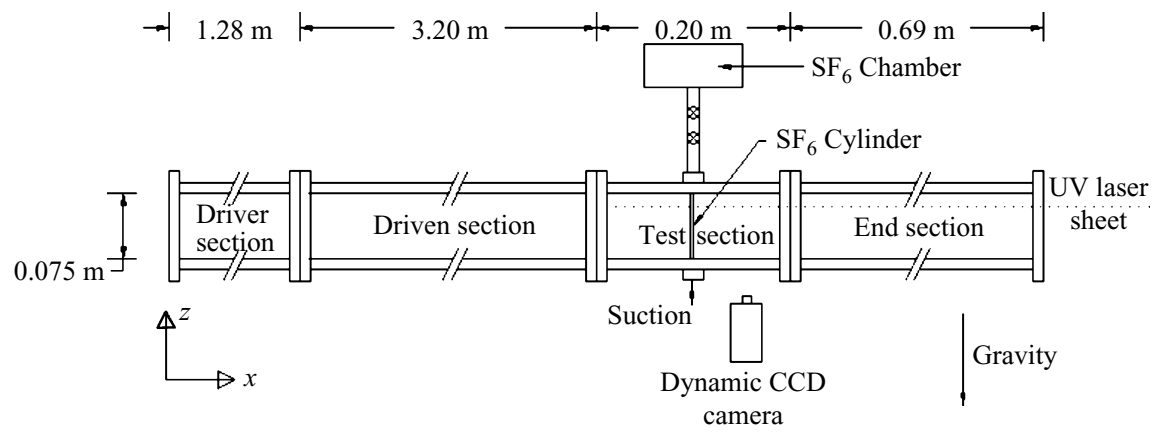

FIGURE 1. Side-view schematic of LANL shock tube experiment.

for the classical case is not applicable due to the circular cross-section of the cylinder, key similarities remain. A baroclinic deposition of vorticity drives the flow, in this case creating two regions of opposite-sign vorticity that distort the cylinder and eventually evolve into a counter-rotating vortex pair (or, to be more precise, counter-rotating vortex columns). Both shear-driven (e.g. Kelvin-Helmholtz) and baroclinic secondary instabilities form, breaking down the structure into smaller scales and further distorting the interface. Also, depending on the Reynolds number of the flow, the vortex cores may transition to turbulence at late times. The wealth of relevant physics arising from a relatively simple initial condition have led to analytical (e.g. Picone \& Boris 1988; Samtaney \& Zabusky 1994), numerical (e.g. Quirk \& Karni 1996), and experimental (e.g. Haas \& Sturtevant 1987; Jacobs 1992, 1993) investigations of this problem.

One of the principal goals in investigating RMI is obtaining an understanding of the resultant mixing. In a simplified view, mixing can be thought of as a two-stage process, the first stage relating to the generation of interfacial area between two fluids and the second stage relating to diffusion across that interface. Thus, mixing may be enhanced by the motions of the flow in several ways. In terms of increasing the interfacial area, the motions may stretch and/or fold the fluid elements along the interface, and the rate of increase may be enhanced by the development of smallerscale motions arising from secondary instabilities or turbulence. Flow dynamics may also enhance the diffusive aspect of mixing, increasing the concentration gradient by straining the interface so that high compressive strain occurs in the direction of the concentration gradient vector. It will be seen from the data that all of these elements play a role in the present experiment. The mixing of passive scalars in complex flows is a rich topic, as the result is highly dependent on the dynamics at all scales. The issue is further complicated in the current problem by the coupling of the scalar field into the dynamics via the baroclinic term in the vorticity equation.

Detailed mixing studies have typically been performed on canonical flows such as turbulent jets and shear layers. Scalar dissipation rate measurements based on planar (two-dimensional) data were performed by Buch \& Dahm $(1996,1998)$ to investigate the fine-scale structure of scalar mixing in turbulent shear flows at high $(S c \gg 1)$ and moderate $(S c \approx 1)$ Schmidt numbers. In both regimes it was found that strained, laminar, sheet-like diffusion layers comprised much of the scalar dissipation rate fields. Scalar concentration measurements were obtained by Su \& Clemens (2003) in turbulent jets using two parallel laser sheets with Rayleigh scattering and laser-induced fluorescence. Here the spatial derivative of the scalar field was computed in all three directions, permitting more accurate estimation of the scalar dissipation rate field. 
These types of highly detailed studies that focus on the physics of mixing, however, are largely absent in the context of shock-induced mixing problems. The Jacobs (1992) planar laser-induced fluorescence (PLIF) visualization study is a careful examination of mixing in a similar problem with discussion of relevant mechanisms. Here the area surrounded by a contour is tracked as a function of time and the change in this area with time is used as a measure of mixing; it decreases monotonically with time as the cylinder mixes with the surrounding air. Jacobs observed the effects of gradient intensification, though his light-gas (helium) cylinder evolves differently from the structure in the present work, and his observations span a pre-turbulent regime, so secondary instabilities are not observed. Zhang et al. (2004) performed a comparison between computational and experimental results for the single-cylinder problem with $\mathrm{SF}_{6}$ as the heavy gas. The authors used histograms of $|\nabla \rho|$ to obtain a global estimate of gradient intensification in the problem, showing that $|\nabla \rho|$ increases from the initial condition at two later times in their simulations; they noted that understanding this phenomenon is crucial to understanding mixing. Zhang et al. also observed that this density-gradient steepening will feed back into the dynamics of the flow through the baroclinic term and in fact leads to increased secondary baroclinic vorticity production, which at intermediate times becomes significant in regions of high acceleration (where $\nabla p$ becomes significant in addition to the enhanced $\nabla \rho$ ). Kumar et al. (2005) performed an experimental study of shock-induced mixing using PLIF visualization for several initial configurations of gaseous inhomogeneities. They examined one aspect of mixing at early time, namely the stirring of the fluid by the primary instability. Stirring was measured by tracking the growth of interfacial material lines with time. The lengths of these material lines were observed to grow exponentially prior to transition of the flow.

In the present work, we perform a detailed quantitative examination of shockinduced mixing processes by estimating the scalar dissipation rate experimentally for the first time in a shock-accelerated flow. High signal-to-noise ratio measurements of the concentration field are obtained using quantitative planar laser-induced fluorescence, from which the mixing rate data are computed. The data show the spatially varying instantaneous mixing rate as a function of time, and thus permit a detailed examination of the physics of mixing in the flow. We examine the contributions of different regions of the flow to the overall mixing rate, consider the relative contributions of primary and secondary instabilities, and probe the underlying mechanisms responsible for the enhanced mixing after shock passage.

\section{Experiment}

A side-view schematic of the LANL shock tube experiment is shown in Figure 1. A thorough description of the operation may be found in previous papers (Rightley et al. 1999; Prestridge et al. 2000; Tomkins et al. 2003), but the basics will be briefly reviewed here. Air is released from the pressurized driver section by puncturing a membrane, from which a Mach 1.2 shock forms (in air) that interacts with the dense gas $\left(\mathrm{SF}_{6}\right)$ in the test section. In the present experiment the dense gas is in the form of a vertical cylinder, created using the membraneless approach pioneered by Jacobs (1992), which involves flowing the gas downward through a circular nozzle (with diameter $D=5 \mathrm{~mm}$ ) under gravity. Exit velocity is approximately $20 \mathrm{~cm} \mathrm{~s}^{-1}$, chosen as the highest velocity for which the cylinder flow is completely steady throughout the test section (to minimize diffusion but ensure shot-to-shot repeatability). 
Concentration (mass-fraction) maps of the dense gas are obtained using planar laser-induced fluorescence (PLIF) with acetone as a tracer (Lozano, Yip \& Hanson 1992). The acetone is excited using a dual-head, frequency-quadrupled Nd:YAG laser from New Wave Research that produces roughly $20 \mathrm{~mJ}$ per pulse @ $266 \mathrm{~nm}$. Two dynamic images are captured during each experimental realization by a cooled, back-illuminated, 16-bit CCD from Apogee Instruments. The CCD chip contains $1024 \times 1024$ square pixels, each measuring $24 \mu \mathrm{m}$ in dimension, which corresponds to $48 \mu \mathrm{m}$ pixel spacing in the object plane with a field of view of $49.2 \mathrm{~mm}$. The images are collected through a Tamron SP Macro Zoom lens with f/2.8. Numerous experiments are performed to build up a set of dynamic data with 11-20 realizations at each of 10 times: $t=130,220,310,400,490,560,650,760,880$, and $1000 \mu$ s after shock impact. (The slightly non-uniform $\Delta t$ between timings is necessary to ensure the capture of whole structures within the field of view at each of the two camera positions used.) The initial condition is also captured on each experimental run approximately $1 \mathrm{~s}$ before shock impact and imaged using an intensified 8-bit camera from Hadland Instruments (with pixel size of $20 \mu \mathrm{m}$ projected into the object plane, although true resolution is lower due to image blur).

Potential sources of error were systematically eliminated or mitigated wherever possible with the goal of obtaining quantitative concentration maps. In processing the data, we removed effects from the camera flatfield (i.e. the response to a uniform light source, including low-frequency lens effects and high-frequency pixel-to-pixel variation) and the camera darkfield (i.e. the bias offset) by explicitly measuring each and using the resulting fields in later solving for the concentration. Images of background intensity were also obtained for each experimental configuration on each day. Typically 10 images were captured and averaged to yield an estimate of background light for each dataset.

The spatial variation of the light sheet was measured by filling the test section with an acetone/air mixture and moving the laser-access window upstream, adjacent to the dynamic measurement area. (If the window is left in place the acetone mixture in the end section completely absorbs the beam before it reaches the test section.) Light-sheet calibration images were then acquired for each laser, on each day of experimental runs, immediately following the dynamic experiments. These calibration images were obtained by integrating over 30 laser pulses to minimize noise, and separate background images were obtained for each set of calibration images with a matching exposure duration. The calibration images were then processed using the camera flatfield, camera darkfield, and background-intensity images, yielding the fluorescent intensity that contained the laser-sheet spatial variation information. These processed intensity fields, however, still exhibited the expected exponential decay (Beer's law) of the signal with distance, due to absorption of the beam by the acetone mixture. These absorption effects were removed by fitting an exponential curve along the direction of propagation and normalizing the image intensity along each ray by the curve value. The resulting fields are thus estimates of the true spatially varying light-sheet intensity for each laser, on each day, and are used in removing this effect in the dynamic images.

The fluorescent tracer was introduced by bubbling the heavy gas through liquid acetone. The acetone bubbler was immersed in a constant-temperature bath, to minimize effects of temperature on the acetone absorption and subsequent fluorescence, and the bubbler fluid level was monitored to stay in a range in which no variation in intensity was observed with fluid level (based on a separate study). The effects of laser pulse-to-pulse variation are removed using conservation of mass, as the 
entire structure is within our field of view at all times and the cylinder flow is extremely steady. The laser pulse intensity was maintained below approximately $13 \mathrm{~mJ}$ per pulse using a beam splitter, as this is the level above which we began to observe saturation of the signal in a separate study (i.e. above this level we no longer observed the required linear response between fluorescent signal and incident intensity). Absorption of the laser sheet as it propagates through these thin structures is considered negligible throughout most of the data-the maximum effect is expected along the centreline of the initial condition and is estimated to be $1.8 \%$. The fluorescence occurs on very short time scales $(\approx 10 \mathrm{~ns})$, yielding an effectively instantaneous measurement, and the longer-time-scale phosphorescence signal is quenched by a small amount of oxygen present in the cylinder due to diffusion as it flows.

Three sources of error could not be removed, however. Shot noise due to the stochastic nature of photon arrival creates a random error of $\sqrt{N}$, where $N$ is the number of incident photons. In a region of the present data in which $c=50 \%$ of the peak concentration, this error is $\pm 2 \%$. Also, the data processing revealed that small-scale, high-frequency variations occurred in the laser light sheet between the acquisition of calibration images and the dynamic data (even though calibration images were obtained each day). These variations are seemingly random and thus treated as a random error, and were found to be approximately $\pm 4 \%$ of the signal. Finally, there is an error associated with the fact that the presence of acetone makes this problem a three-component mixture, although two of the components are well mixed before being introduced to the third (air). One implication of this is that a slight relative diffusion occurs between the acetone and the $\mathrm{SF}_{6}$ during the roughly $0.1 \mathrm{~s}$ it takes for the mixture to reach the measurement plane (located $2 \mathrm{~cm}$ below the nozzle). Our estimate of the air- $\mathrm{SF}_{6}$ molecular diffusion coefficient is $\mathscr{D}=0.98 \times 10^{-5} \mathrm{~m}^{2} \mathrm{~s}^{-1}$, while the lower molecular weight of the acetone yields a slightly higher coefficient for air-acetone, $\mathscr{D}=1.21 \times 10^{-5} \mathrm{~m}^{2} \mathrm{~s}^{-1}$. The difference in resulting diffusion length scales at the time of interest is approximately $200 \mu \mathrm{m}$ for the two gases, suggesting that the acetone tracer has diffused slightly more at a given radial location than the $\mathrm{SF}_{6}$ at the time of shock impact. Although a diffusion length-scale difference would only shrink as the cylinder is stretched, and differential diffusion would not be significant after shock impact, this effect could bias estimates of absolute mixing rates. In the present paper, therefore, the conclusions focus on the relative mixing rates in different regions of the flow, which are not expected to be significantly modified by this effect.

The raw intensity images were processed to solve for the volume fraction $\left(c_{v}\right)$ of the dense gas. Mass fraction $(c)$ of the $\mathrm{SF}_{6}$ was computed from the volume fraction, and will be the scalar of choice in the present work due to its appearance in the definition of scalar dissipation. The resulting concentration maps were then low-pass filtered with a small Gaussian kernel to reduce high-frequency noise. The Gaussian kernel size was chosen to yield $50 \%$ reduction in the signal power spectrum at the measurement Nyquist frequency, which corresponds to a wavelength of twice the pixel spacing, $\lambda_{n y q}=2 \Delta x$.

The stated resolution of the dynamic camera $(\Delta x=\Delta y=48 \mu \mathrm{m})$ is defined as the pixel size projected into the object plane, and images of resolution (Air Force) targets confirm that this is the true resolving capability of the imaging system. The structure is nominally two-dimensional, with any variations in $z$ expected to be of much larger scale than our out-of-plane resolution of $\Delta z \approx 200 \mu \mathrm{m}$, defined by the maximum light sheet thickness within the field of view. This estimate of maximum light-sheet thickness is based on geometrical considerations of the beam as it is focused down in $z$; we 

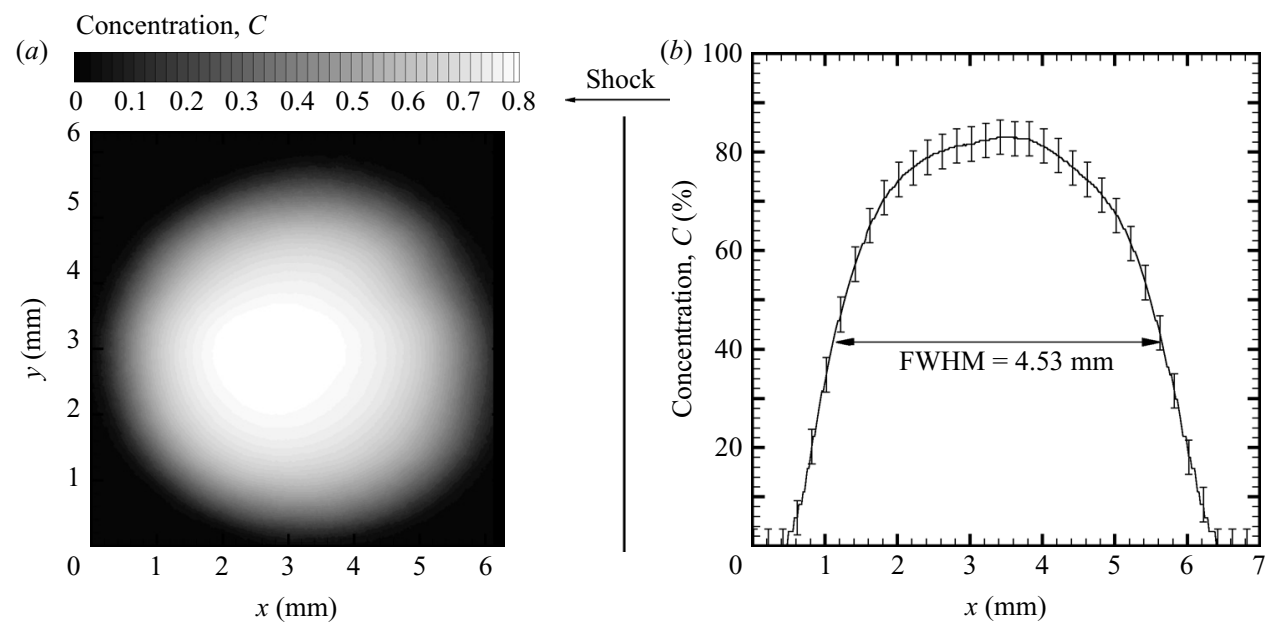

FIGURE 2. Initial condition. (a) Ensemble-averaged concentration of heavy gas with image contrast $c=[0,0.80]$ and shock moving from right to left. $\left(c_{\max }=0.84\right)$. (b) Lineout through centre of image showing FWHM. Error bars are $\pm \sigma$, one standard deviation in the ensemble. Nozzle size is $D=5.0 \mathrm{~mm}$.

also estimate the minimum light-sheet thickness as the size of the diffraction-limited waist, $\Delta z_{\min } \approx 160 \mu \mathrm{m}$ (see e.g. Adrian 1991).

In estimating scalar dissipation rate, resolving concentration gradients is critical. Given the significant energy input in the current problem and the late-time instability observed in certain regions, the smallest velocity gradient in the flow at late time within turbulent regions is expected to be on the order of the Kolmogorov scale, roughly $10 \mu \mathrm{m}$. The smallest concentration gradient of the flow might be of comparable scale (with $S c \approx 1$ ), and therefore sub-resolution, if the initial condition were sharp. We are able to resolve the concentration gradients, however, due to the diffuse nature of the initial condition: the structure is rapidly strained, but initially diffuse, and so the gradients, although steepened, never approach the pixel spacing in scale. The smallest observed gradient scales are approximately 4-5 pixels, based upon one-dimensional profiles through the finest structures. Note that such a scale does represent significant gradient steepening relative to the initial condition, in which the full-width at halfmaximum (FWHM) of the structure is approximately 94 pixels across (in terms of the dynamic camera resolution). At late time, it is possible that the vortex cores are becoming turbulent, with associated creation of small-scale structures in the velocity field. Due to the consistent diffusion taking place, however, the scales of the concentration field appear to be much larger - on the order of a characteristic diffusion length scale, for example, estimated to be $L_{d} \approx 250-500 \mu \mathrm{m}$ at late time. Hence, the present resolution of $48 \mu \mathrm{m}$, while insufficient to resolve all concentration gradients in many high-Reynolds-number mixing flows, appears to be sufficient for the problem at hand.

\section{Concentration fields}

\subsection{Initial condition}

The initial concentration (mass fraction) distribution of the gas cylinder is shown in figure 2. Figure 2(a) shows the ensemble-averaged concentration field for all of the 
shots included in this study. Note that although variations in spatial location of the steady column are expected to be minimal before the shock, the ensemble averaging is performed using an iterative, correlation-based procedure (CBEA, Tomkins et al. 2003) that removes any effects in the average from slight misalignment of the imaged cylinders by effectively registering each image before averaging. The average initial condition is not perfectly axisymmetric, but quite close.

The absolute concentration level in the cylinder is estimated in two ways. At the time of the experiment, the peak concentration was estimated based on simulations of the steady flowing cylinder performed at the University of Chicago ASCI Flash centre (Weirs, Dwarkadas \& Plewa 2004). In these simulations, inlet velocity and concentration are specified and the concentration evolves in time in the geometry of the shock tube, resulting in a steady flow with peak concentration (mass fraction of $\mathrm{SF}_{6}$ ) at the light sheet location of $c_{\max }=0.83$. Comparison between the experimental and computational concentration profiles shows good agreement in terms of material distribution.

Subsequently, an experimental measurement of peak concentration was performed using a calibration procedure developed after the initial set of experiments. This procedure uses a source of pure $\mathrm{SF}_{6}$-acetone mixture placed at the light sheet location, allowing comparison of the fluorescence signal with that obtained using the nominal experimental configuration (i.e. with attendant diffusion). This measurement yielded a peak concentration of $c_{\max }=0.84$, in excellent agreement with the estimate from the simulation.

A lineout through the centre of the image is shown on figure $2(b)$, revealing the FWHM of the initial condition to be $4.53 \mathrm{~mm}$. It is notable that the ensemble averaging has damped whatever noise was present in the individual images, resulting in a profile that, for an experimental measurement, is very smooth. A measure of the shot-to-shot variation is captured by the error bars included on the plot, which are of magnitude $\pm \sigma$, where $\sigma$ is one standard deviation of the realizations in the ensemble.

\subsection{Dynamic concentration maps}

Dynamic data are presented in figure 3 , which shows concentration maps for ten times after shock passage. The figure is created from several experimental runs, with high repeatability observed on the larger, more deterministic scales and greater shot-to-shot variability observed in more stochastic regions, e.g. unstable regions at later times. Although the exact appearance of these stochastic regions will vary, at each time the presented image is representative of the data set as a whole. Several previous studies have imaged shock-accelerated gas cylinders (e.g. Jacobs 1992; Tomkins et al. 2002), discussing the primary instability and prominent features in the evolution, so these aspects will only be reviewed briefly here. The primary instability is associated with the deposition of two blobs of opposite-sign vorticity on the edges of the cylinder (left and right edges in figure 3). The vorticity begins to distort the dense gas, stretching the structure and eventually rolling up into a counter-rotating vortex pair. The length and width of the structure grow in time, as does the vortex spacing. Eventually certain regions of the structure begin to show evidence of further instability and transition to turbulence.

The high resolution and high signal-to-noise ratio of the present data permit the clearest experimental visualization of this transitional process obtained to date. At $t=560 \mu$ s, evidence of secondary instabilities (slight waviness) appears on the outer edges of the structure. As time progresses, these perturbations grow in regions of high shear and indeed look quite like Kelvin-Helmholtz $(\mathrm{K}-\mathrm{H})$ rollers (e.g. along the 


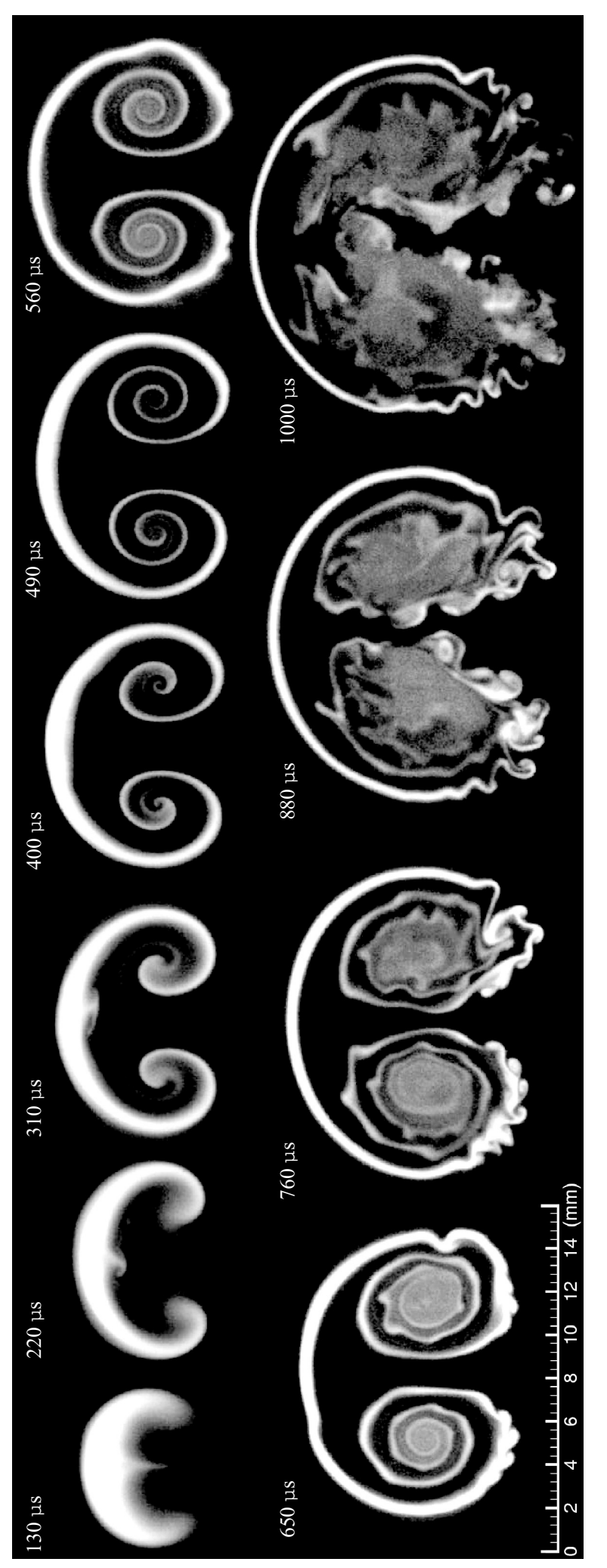

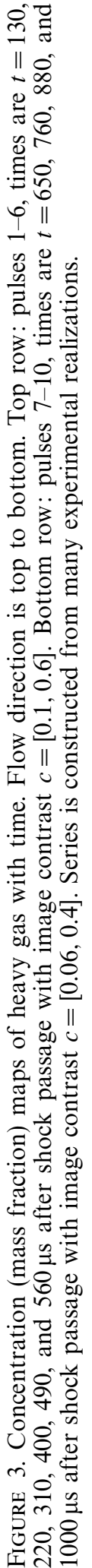


lower edges in pulses 7-9). Within the vortex cores, instabilities are clearly visible at $t=650 \mu \mathrm{s}$, although here there is some debate as to the nature of the instability. There is baroclinic generation of vorticity due to the radial pressure gradient created by rotation (Peng, Zabusky \& Zhang 2003), but also one $\mathrm{SF}_{6}$-air interface will be $\mathrm{R}-\mathrm{T}$ unstable. These phenomena are probably complicated by shear as well. Within the cores at $t=880 \mu \mathrm{s}$, for example, are features emerging from the heavy gas that appear to be bent over by shear. Regardless of the relative contribution of these secondary instabilities, however, it appears that they rapidly cause transition in the flow once they begin to grow. The vortex cores and $\mathrm{K}-\mathrm{H}$ regions of the flow undergo a very rapid transition between, for example, pulses 8 and 10, evolving from an unstable but relatively coherent state (pulse $8, t=760 \mu$ s) to a well-mixed and incoherent state (pulse $10, t=1000 \mu \mathrm{s}$ ) in a matter of $\approx 240 \mu \mathrm{s}$. Thus, the visualizations strongly suggest that secondary instabilities play a key role in the mixing process. The scalar dissipation rate measurements presented next confirm that a high mixing rate is associated with these structures. We will also see the somewhat counter-intuitive result that the bridge-the upper region that connects the two halves of the structures - contributes significantly to the instantaneous mixing rate as well.

\section{Scalar dissipation rate fields and mechanisms of mixing}

Our goal in the present work is a detailed examination of the mixing processes in this problem. The quality of the data permits experimental estimation of the instantaneous scalar dissipation rate (or mixing rate), $\chi(\boldsymbol{x}, t) \equiv \mathscr{D}(\nabla c \cdot \nabla c)$, from the concentration fields $c(\boldsymbol{x}, t)$, which provides quantitative information on the spatial distribution of the mixing at each time. Here $\mathscr{D}$ is the molecular diffusivity between the gases, estimated to be $\mathscr{D}=0.98 \times 10^{-5} \mathrm{~m}^{2} \mathrm{~s}^{-1}$ for air- $\mathrm{SF}_{6}$. Mathematically, the term arises in simple manipulation of the advection-diffusion equation for a scalar, appearing as a sink term in an expression for the evolution of the scalar 'energy' $c^{2}$ (see e.g. Su \& Clemens 2003). Scalar dissipation measurements have been used to probe fine-scale mixing in canonical turbulent flows (Buch \& Dahm 1996, 1998), constrain combustion models with turbulent jets (Su \& Clemens 2003), and understand passive-scalar mixing in isotropic turbulence (Eswaran \& Pope 1988; Girimaji 1992). We compute $\chi$ from the scalar fields using the numerical approach described in Buch \& Dahm (1996). This approach relies on the eight surrounding points for a given pixel, computing one estimate of $\chi$ based on the four points aligned with the grid, computing a second estimate based on the four corner points (those aligned with a grid rotated $45^{\circ}$ ), and averaging the two values. Our estimate is based on the two in-plane components of the concentration gradient.

\subsection{Instantaneous mixing rate maps}

Spatial maps of the normalized mixing rate are shown in figure 4 for the same experimental realizations presented in figure 3 . In these data the spatially varying instantaneous rate of mixing is easily visualized. At early times, total mixing increases, with the vortices from the primary instability stretching the material and steepening concentration gradients. At intermediate to later time, secondary instabilities develop and are associated with regions of high $\chi$, particularly the $\mathrm{K}-\mathrm{H}$ structures along the lower edges of the cylinder, as might be expected based on inspection of the concentration data. Within the vortex cores, the implications of the mixing rate data are interesting. The mixing rate appears to peak before the onset of secondary instabilities, at $t=490 \mu \mathrm{s}$, as heavy gas is continually stretched around the vortex cores, increasing interfacial area and steepening concentration gradients along that interface. 

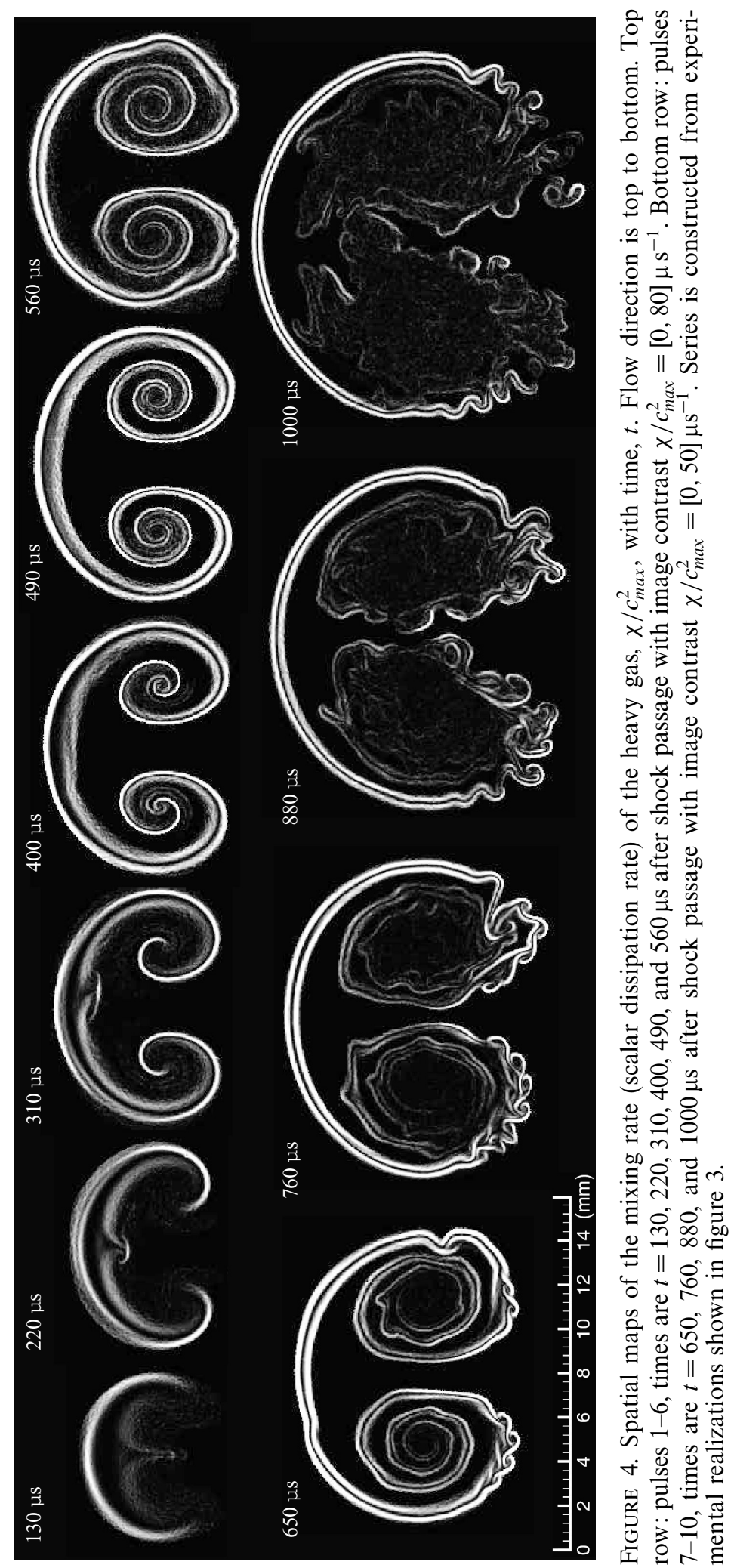
Moreover, at late times - when secondary instabilities are transitioning the flow - the cores contribute very little to the mixing, as although there are no doubt small-scale velocity gradients present to drive mixing, the fluid there is already in a well-mixed state. Hence, it appears that the vortices associated with the primary instability accelerate mixing in the vortex cores via simple interfacial stretching before the secondary instabilities effectively transition the cores, although once transition occurs in these regions, the smaller velocity gradients rapidly eliminate whatever concentration gradients remain. In this sense, the energy deposited by the primary instability gives birth to two competing mechanisms around the cores: one, simple stretching by the deposited vorticity, and another, the development of secondary instabilities. The former impacts the scalar field almost immediately, but ultimately cannot mix as efficiently as turbulence. The latter is simply not present until instabilities have had time to develop but, once present, would be expected to mix extremely efficiently.

One region that is associated with a high level of instantaneous mixing throughout the duration of the experiment is the bridge, the non-turbulent band of fluid connecting the two halves of the structure. The bridge appears to be a region of intense mixing from around $t=400 \mu$ s on, whereas other transitional regions of the flow, such as the vortex cores, exhibit a rapid increase in mixing to intermediate times but then fall off sharply (at later times). In the bridge region, the large-scale strain field created by the two vortices (associated with the primary instability) rapidly stretches the interface, consistently steepening the concentration gradients such that substantial mixing appears to occur in the bridge over the duration of the measurement.

\subsection{Total mixing rate}

The total mixing rate is denoted $\langle\bar{\chi}\rangle$, where the overbar represents spatial integration in $x$ and $y$ over an instantaneous realization and the angle brackets represent an average over all realizations for a given time. $\langle\bar{\chi}\rangle$ is plotted as a function of time in figure 5(a), including error bars of $\pm \sigma$, where $\sigma$ is one standard deviation of the $\bar{\chi}$ values at each time. The total mixing rate is observed to rapidly increase up to $t=490 \mu \mathrm{s}$ and then remain roughly constant for $\approx 300 \mu \mathrm{s}$. The mixing rate then decreases at late time, despite the fact that the flow is transitioning, as certain regions have become well-mixed. (Note that pulses 9 and 10 are within the error associated with statistical variation between realizations at a given time $( \pm 2 \sigma)$, so no significance is attributed to the slight increase at pulse 10.) The global mixing rate includes contributions from several mechanisms, of course, including the steepening of concentration gradients through straining, an increase in interfacial area due to stretching by the large-scale strain field (observed in the cores at early times, for example), and an increase in interfacial area due to the presence of secondary instabilities and associated small-scale velocity gradients (folding/stretching the interface locally). Isolating the contributions from any one of these mechanisms is not typically possible with concentration data. The bridge region provides a unique opportunity to do so, however, as one mechanism (gradient steepening via large-scale straining) is dominant in the mixing process. As mentioned earlier, Jacobs (1992) discussed this mechanism in the context of a similar problem, and Zhang et al. (2004) have observed a global increase in density gradients with time in a similar flow. Here we aim to better understand the fundamental mixing processes, and although this mechanism will certainly be present elsewhere in the flow, by isolating and quantifying the mixing in the bridge we obtain a lower bound on the contribution of gradient intensification via straining to mixing in the flow.

Masks are used to isolate the bridge region and determined for each realization. Masks are created from the data using a broad filter and threshold to define a region 

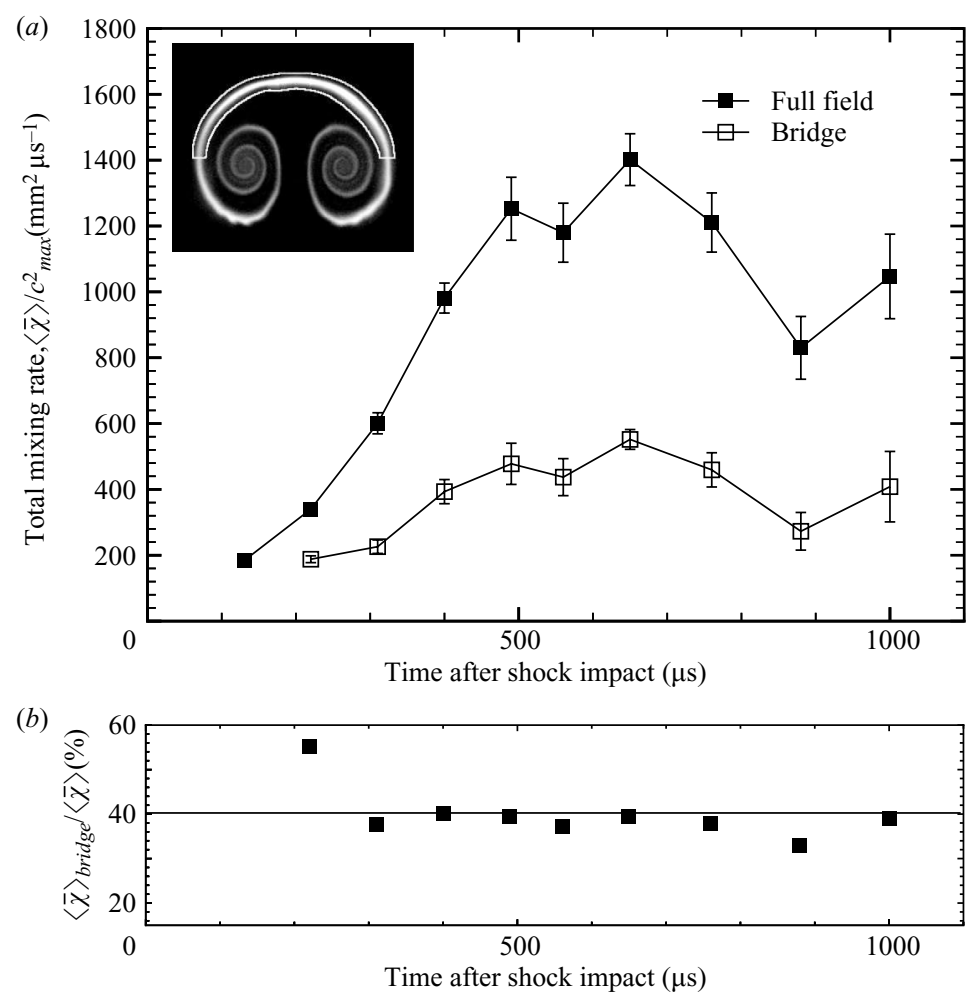

FIGURE 5. (a) Total mixing rate, $\langle\bar{\chi}\rangle / c_{\max }^{2}$, as a function of time for the shock-accelerated gas cylinder. Solid symbols, total mixing rate for the flow. Hollow symbols, bridge contribution to total mixing rate. Error bars are $\pm \sigma$, where $\sigma$ is one standard deviation of $\bar{\chi}$ values for a given time (see text). Also shown in the inset is the edge of a sample mask used to extract the bridge contribution, overlaid on the corresponding concentration field. (b) Lower bound on the contribution of gradient intensification by straining to mixing: percentage of the total mixing rate in the bridge. Horizontal line is $40 \%$.

within which high signal levels will be contained. This region is then cropped with a horizontal line at the point above which the effects of secondary instabilities were not observed, which is roughly the point at which the material interface becomes vertical (i.e. aligned streamwise). An example is shown in the inset of figure 5(a), where a mask boundary is overlaid on the corresponding concentration field. In estimating the contribution to mixing from the bridge, $\chi$ values were simply integrated within this region, and then averaged over all realizations. Again, summing within a mask such as this provides a lower bound on the quantity of interest.

The results are plotted in figure 5(a) (hollow symbols). (No contribution is given for pulse 1, at which time a bridge is not easily defined.) Interestingly, the trend of the bridge contribution follows the total mixing rate quite closely, with an increase at early time, a rough plateau, and a decrease at later times. The percentage contribution of the bridge to the total mixing is shown in figure $5(b)$. The bridge contributes a significant and nearly constant percentage of the total mixing from $t=310 \mu$ s onwards, around $40 \%$. Thus, despite the more obvious role of secondary instabilities in the mixing process, it is seen that the primary instability, through the large-scale strain field that it creates, contributes significantly to the mixing at all times via intensification of concentration gradients. Note that the mask effectively isolates this particular 
mechanism not only by removing the $\mathrm{K}-\mathrm{H}$-unstable regions and vortex cores, but also by considering a roughly constant area (and thus removing the effects of increased interfacial area by stretching, which is also associated with the primary instability in some regions).

It should be noted that there exists a bias in our assessment of the bridge contribution to mixing relative to other regions of the flow. This bias arises from our reliance on the two in-plane components of the concentration gradient for the $\chi$ estimate. As mentioned earlier, this estimate should be adequate for this nominally two-dimensional flow. In any turbulent flow regions, however, out-of-plane gradients are expected to be of the same order as the in-plane gradients, and so two-dimensional estimates are probably biased low. In the present experiment, we do not have velocity information to quantitatively assess transition, although the vortex cores clearly become unstable at intermediate times and the flow is quite possibly three-dimensional in the cores at late time. Hence, our estimate of the total scalar dissipation at late time in the vortex cores is possibly biased low. As the bridge estimate is given as a percentage of the total mixing, this effect would bias the bridge estimate high. Defining a vortex core boundary or a turbulent/non-turbulent boundary is difficult to do objectively, but estimates based on a subjective boundary suggest that the vortex core contribution to mixing at late time $(t=880 \mu \mathrm{s})$ is $15 \%$. If one applies a correction to this (twocomponent) figure assuming isotropy for local gradients - which is the case of greatest possible error associated with this effect - then the vortex cores would contribute in excess of $20 \%$ of the total mixing at late time. With this correction (again, applicable only if the vortex cores have become three-dimensional) the bridge contribution would be reduced by approximately $3 \%$ from the value of $40 \%$ stated above.

There is a roughly constant plateau observed at intermediate times (in the approximate range $450<t<800 \mu \mathrm{s}$ ) for the bridge mixing rate, which is noteworthy considering that material is consistently being transported through the ends of the mask. The presence of this plateau suggests that the bridge flow is in an approximately steady state, with the effects of diffusion and compressive strain in balance, as in a typical strain-limited diffusion layer found in canonical turbulent mixing flows. Thus, due to the large-scale nature of the strain field relative to the concentration gradients, this layer may be similar to the diffusion layers found on the smallest scales of the flow in high-Sc turbulent mixing (as in Buch \& Dahm 1996 for example).

Note that gradient intensification will certainly be present (and will probably play a significant role in mixing) in flows with a variety of initial conditions, e.g. along the sides of strained bubbles and spikes in classical single-interface $\mathrm{R}-\mathrm{T}$ and $\mathrm{R}-\mathrm{M}$ flows. The evolution at early to intermediate times in $\mathrm{R}-\mathrm{M}$ flows in particular tends to be dominated by the deposited vorticity. Hence, the results here are representative of the general case of $\mathrm{R}-\mathrm{M}$ flows with initial-condition perturbations of relatively large scale (meaning larger than the diffusive scales of the post-shock flow). In this regime, one would expect the scale of the deposited vorticity to be correspondingly large, at least in some dimension. This would lead to an evolution of the flow (before any transition to turbulence) that is dominated by a large-scale vorticity field operating on material interfaces established in the initial conditions, a situation in which mixing would be driven by stretching and gradient intensification via straining, as observed in the present flow.

\subsection{Estimate of a mixing time scale}

The total mixing rate curve also shows a region of roughly constant values over the approximate time range $450<t<800 \mu$ s. In his investigation of a light-gas cylinder, 
Jacobs (1992) measured the area contained within the $50 \%$ concentration contour as a function of time, and used the time rate of change of this area to estimate mixing rate. He obtained a collapse of mixing data by non-dimensionalizing using a time scale based on a simple model proposed by Marble (1985). The model was developed for combustion applications but considered the fundamental problem of a diffusive interface strained by a potential vortex, and led Jacobs (1992) to propose a mixing time scale of

$$
t_{m}=\frac{r^{2}}{\Gamma^{2 / 3} \mathscr{D}^{1 / 3}},
$$

where $\Gamma$ is the circulation of the vortex and $r$ is the distance from the vortex to an annulus of material being mixed. Here we take $r=D$, the cylinder diameter, which is a reasonable approximation of the distance between the vortex cores and the mixing regions. $\Gamma$ for the current flow may be estimated by interpolating between experimentally measured values of circulation (obtained with PIV) for different size $\mathrm{SF}_{6}$ cylinders (Tomkins et al. 2003). For the two cylinder sizes for which PIV data are available $(D=3$ and $8 \mathrm{~mm})$, measured circulation is exactly proportional to diameter, which makes physical sense given that the diameter is proportional to the circumference, along which vorticity is deposited. Linear interpolation between these values for a $D=5 \mathrm{~mm}$ cylinder yields $\Gamma \approx 0.375 \mathrm{~m}^{2} \mathrm{~s}^{-1}$. The resulting mixing time scale is $t_{m} \approx 2.2 \mathrm{~ms}$, and so our data extend to approximately $t / t_{m}=0.45$. (It is worth noting that the choice of length scale is somewhat subjective here, and other reasonable choices exist that would yield somewhat different values of $t_{m}$ and $t / t_{m}$.) Jacobs' results were focused on a slightly shorter time range, by this time scale, extending to $t / t_{m}=0.36$. It is interesting to note that Jacobs observed a region of approximately constant mixing rate-a range where the slope of the contour area was non-zero and approximately constant - that occurred over a time range of roughly $0.10<t / t_{m}<0.25$. In the present data, the roughly constant plateau region occurs over the non-dimensional time range of $0.20<t / t_{m}<0.36$, which overlaps the range observed by Jacobs. It is difficult to conclude from this degree of agreement, however, that the same physics are at work, due to important differences in the two datasets. Jacobs' light-gas cylinder forms a different structure when shocked than the present structure, and the data cover a pre-turbulent range, so that secondary instabilities are not observed to develop. The curve in figure 5, by contrast, is a reflection of several underlying mechanisms, including secondary instabilities that form and rapidly develop during the time over which this plateau region occurs. Thus, the observed plateau in the present work may simply be the result of a sum of contributions from different mechanisms that vary individually with time but whose sum happens to stay roughly constant over the observed range.

\subsection{Evolution of scalar gradients}

The plateau observed in the mixing rate data for the bridge region does not suffer from this complication. Careful inspection of the scalar dissipation rate maps suggests, however, that the bridge reaches this state of rough equilibrium much more quickly at the upstream edge than at the downstream edge. At early to intermediate times, the upper edge appears to quickly sharpen, developing an increased rate of mixing in a short time. By contrast, the lower (downstream) edge is much more diffuse early, although the profile of the lower edge sharpens at approximately $t=560-650 \mu \mathrm{s}$ and it begins to contribute more substantially to the mixing. Vertical profiles of the mixing rate through the bridge region would provide a clear and more quantitative view of the relative contributions of the upper and lower edges. We are interested in the average 


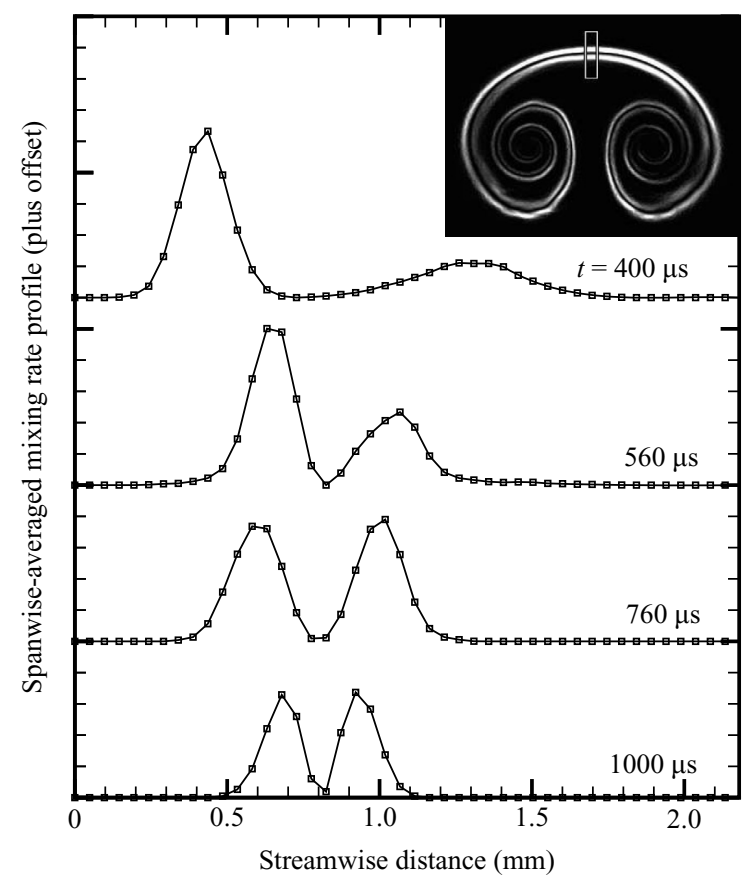

FIGURE 6. Spanwise-averaged profiles of the mixing rate based on an ensemble average, $\chi_{e} / c_{\max }^{2}$, as a function of streamwise location. Inset figure shows $\chi_{e}(\boldsymbol{x}, t=560 \mu \mathrm{s})$. Profiles intersect the bridge and represent spanwise (horizontal) averages over the region defined by the box in the inset. Data are offset along the ordinate to more easily view evolution of profile with time.

behaviour of the flow, of course, so rather than extracting profiles from the structures included in figure 4 we will compute and examine the ensemble average. The averaging is performed using the correlation-based ensemble averaging procedure (CBEA, Tomkins et al. 2003) employed for the initial condition data, which removes the spatial jitter of the structures inherent in this experiment through an iterative correlation technique that registers the structures before the ensemble average. Note that a straightforward ensemble average would not yield a physically meaningful result due to the small shot-to-shot variation in the position of the structures on the CCD array (see Tomkins et al. 2003). The CBEA procedure does yield a meaningful average, however, and an estimate of average behaviour of the upstream and downstream edges of the bridge is thus obtained by computing the mixing rate associated with this ensemble average, $\chi_{e}(\boldsymbol{x}, t) \equiv \mathscr{D}(\nabla\langle c\rangle \cdot \nabla\langle c\rangle)$, where $\langle c\rangle$ is the ensemble average of $c(\boldsymbol{x}, t)$ at each time.

Vertical profiles through the region of interest are created from the $\chi_{e}$ data by extracting a region of 50 rows by 11 columns located along the spanwise midplane of the structure and centred on the bridge (an example of this region is represented by the small box in the inset of figure 6 , which shows $\chi_{e}(\boldsymbol{x}, t=560 \mu \mathrm{s})$. Vertical profiles are obtained by averaging spanwise in this region, yielding a one-dimensional function varying in $x$. $\chi_{e}$ profiles for four times are shown in figure 6 , with offsets in the vertical axis added for ease in visualizing the profile evolution with time. The profiles confirm the trends apparent from the $\chi$ images in figure 4 . At $t=400 \mu \mathrm{s}$, the mixing occurring on the upstream edge is substantial, while the downstream edge is much more diffuse and creates a much lower mixing rate, which is only apparent as a smooth bump in the profile. The rapid steepening of gradients along the upper edge 
(relative to the lower edge) is probably associated with the impingement of fluid from upstream-when viewed in the reference frame of the convecting structure-as the structure propagates upstream at $\approx 10 \mathrm{~m} \mathrm{~s}^{-1}$ relative to the shocked air that surrounds it (which is propagating downstream at $\approx 100 \mathrm{~m} \mathrm{~s}^{-1}$ in the laboratory frame). This upstream fluid probably aids the strain field associated with the vortices to maximize compressive strain along the upstream interface at the centre of the bridge. The straining effect by the vortices is quickly manifested on the lower edge, however, as seen in figure 6: this effect becomes apparent at $t=560 \mu \mathrm{s}$, and by $t=760 \mu \mathrm{s}$ the two edges are almost identical in terms of their contribution to mixing. Finally, at $t=1000 \mu \mathrm{s}$, both edges are further sharpened and moved closer together by the straining, though the mixing rate is somewhat reduced, probably due to a decrease in peak concentrations along the bridge.

\subsection{Distribution of the mixing rate}

To further quantify aspects of the mixing processes, we consider histograms of the normalized scalar dissipation rate, shown in figure 7. The figure shows the percentage of area in the flow field that corresponds to a given value of the mixing rate (note that the area is normalized by the total image area, including substantial background with negligible $\chi$ values, and hence the normalized values are relatively small).

For clarity in viewing, the data are separated into two time regimes, with results at early to intermediate times, $t \leqslant 560 \mu$ s, shown on the top row and results at intermediate to late times, $t \geqslant 650 \mu \mathrm{s}$, shown on the bottom row. The figure shows histograms that include values over the entire field (left column) and in the bridge region alone (right column).

Early-time evolution is shown in figure $7(a)$. Here the overall mixing increases in time, with almost all $\chi$ levels becoming more populated. There is a large jump in higher $\chi$ values between $t=310$ and $400 \mu$ s; e.g. the population of $\chi / c_{\max }^{2} \approx 175 \mu \mathrm{s}^{-1}$ values jumps by an order of magnitude, and yet higher values appear for the first time, as the effects of stretching become apparent. For pulses 4-6 the histograms are relatively consistent. There is a slight reduction at pulse 6 from pulse 5, which may be a combination of diffusion catching up with the effects of increased strain and/or statistical sampling error due to the limited number of shots at each time.

The contribution of the bridge region at early time is shown in figure $7(b)$, which also shows a clear increasing trend with time. It is also clear that almost all of the highest values of mixing rate are contained in the bridge: for $\chi / c_{\max }^{2}>200 \mu \mathrm{s}^{-1}$ or so, for example, the histograms look almost identical to those in figure $7(a)$. So the most intense mixing in the flow up to intermediate times is contributed by the bridge region, and thus the most effective mixing mechanism in this problem is gradient intensification via the large-scale strain field created by the primary instability, as discussed earlier. At lower values of the mixing rate the bridge contribution is clearly lower than the total in the flow, so it is for these $\chi$ values that other mechanisms (working in other regions) are also involved.

At later times, shown in figure 7(c), the histograms reflect the general trend of decreasing mixing with time. At $t=650 \mu \mathrm{s}$ the mixing rate is still very high, as seen in previous figures, and the histogram shows that intense mixing (i.e. population at high values of $\chi$ ) is still occurring at this time. The behaviour here is consistent with the results for pulses $4-6$, showing that, like the total mixing rate, the distribution of $\chi$ values is relatively steady over the range $t=400-650 \mu$ s. As time progresses the amount of mixing at all $\chi$ values decreases, with the most obvious reduction at the highest values. The reduction in the histogram levels at lower values is a manifestation 

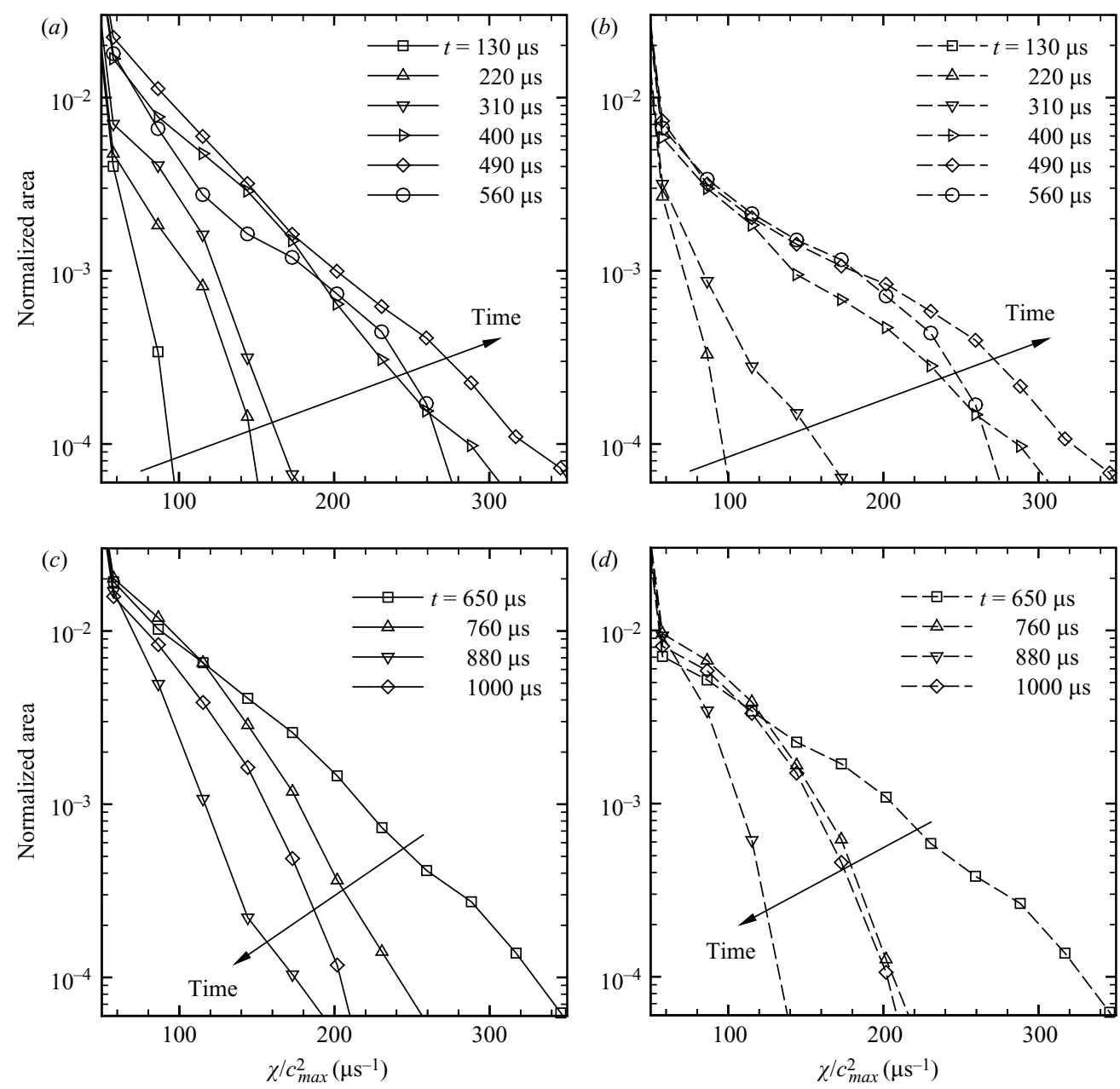

FIGURE 7. Histograms of mixing rate, $\chi / c_{\max }^{2}$. (a) Early to intermediate times; $(b)$ early to intermediate times, bridge region only; $(c)$ intermediate to late times; $(d)$ intermediate to late times, bridge region only.

of the effects seen earlier, namely the cores becoming well mixed and the reduction in the contribution associated with secondary instabilities along the edges of the structure at late times. The bridge also plays a role in this reduction at late time, as evidenced in figure $7(d)$. The dramatic reduction in high $\chi$ values in the bridge after $t=650 \mu$ s is quite striking. It is possible that after this time the advection of material out of the mask lower boundaries is finally having a strong effect on the mixing rate, i.e. the 'supply' of material contained within the bridge is running out, resulting in reduced overall mixing.

\section{Summary and concluding remarks}

An experimental investigation of mixing mechanisms in a shock-accelerated flow is described. The problem considered is a single cylinder of heavy gas in air impacted by a Mach 1.2 shock wave. Planar laser-induced fluorescence measurements are obtained at 10 times after (and at one time before) shock impact, yielding high signal-to-noise 
quantitative concentration maps. The investigation relies heavily on experimental estimates of the instantaneous scalar dissipation rate, computed directly from the concentration fields, which are the first such measurements obtained in a shockaccelerated flow. The instantaneous mixing rate has a clear physical interpretation and proves to be a valuable analytical tool, permitting quantitative evaluation of the physical mechanisms driving the mixing. The spatially varying $\chi$ fields readily reveal a richness of behaviour that is masked by other simpler quantities, such as mixing width.

In this problem, three regions of mixing emerge beyond early times: the bridge, the vortex cores, and the $\mathrm{K}-\mathrm{H}$ secondary instability region. In the first region, mixing is associated with gradient intensification via rapid straining at all times for which a bridge may be defined. Thus, this region provides a unique opportunity to isolate the contributions of a single mechanism. In the vortex core and $\mathrm{K}-\mathrm{H}$ secondary instability regions, mixing is also driven by the large-scale strain field at early times, though here the straining and vorticity create both an increase in surface area (e.g. the spirals developing within the cores) and subsequent gradient intensification along that surface area. At intermediate times, secondary instabilities develop in these regions: $\mathrm{K}-\mathrm{H}$ instability along the outer edges of the flow and a potential mixture of $\mathrm{K}-\mathrm{H}$, $\mathrm{R}-\mathrm{T}$, or baroclinic instabilities within the cores. The scalar field is not entirely passive here: as Zhang et al. (2004) point out, the gradient intensification plays a role in the increased secondary baroclinic vorticity production in these regions observed in simulations. At later times, mixing is primarily associated with an increased surface area due to the stretching and folding of the concentration field by smaller-scale motions that are created by these secondary instabilities.

In visual inspection of the concentration fields the role of secondary instabilities is quite obvious, and may be thought to account for the majority of the mixing. Indeed, it appears from the well-mixed state of the vortex cores at late time that the unstable small-scale structures expected in the cores (associated with the secondary instabilities) are efficient mixers. The scalar dissipation rate results show, however, that gradient intensification via the large-scale strain field contributes significantly to the instantaneous mixing rate, with roughly $40 \%$ of the mixing occurring in the 'bridge' region alone up to $1000 \mu \mathrm{s}$. Moreover, at $t=400 \mu \mathrm{s}$ secondary instabilities are not yet visible in the concentration field - the heavy gas has only been strained by the large scales - and yet the instantaneous mixing rate achieved here is roughly $80 \%$ of the peak values. Plots of the distribution of $\chi$ values further show that the bridge plays a dominant role in contributing the highest values of $\chi$, and hence the most intense mixing in the flow. Thus, the primary instability plays a direct and critical role in the mixing process through the large-scale strain field, in addition to its more obvious role as a source of initial energy.

This quantitative evidence of the crucial role of the bridge in the mixing process appears at first sight to be inconsistent with the unstable nature of the vortex cores, which, as stated earlier, should be highly efficient mixers. These active regions may ultimately be limited in their mixing contribution, however, because they are not sufficiently supplied with fresh (unmixed) fluid on which any small-scale gradients can operate. Thus, although the shock-deposited vorticity greatly enhances mixing in this problem, the total mixing rate is not optimal in the sense that the regions in which fine-scale velocity gradients are expected are spatially distinct from the regions of purest fluid.

The present results have implications for the concept of a mixing transition in unsteady transitional flows. A 'mixing transition', in the sense of Dimotakis (2000), 
implies the existence of a wide range of scales in the flow, as found in a classic cascade. The vortex cores in the present flow are an example of a region that appears well mixed, but it is not obvious that fully developed turbulence is present, in the sense of an established wide range of scales. Thus, it is important to distinguish between a flow being well mixed and a flow having undergone a mixing transition. The former does not necessarily imply the latter, due to the effectiveness of the primary instability in mixing via the mechanisms described herein. If the flow has undergone a true mixing transition, however, implying the existence of a wide range of scales, then indeed it would be expected that the flow would also be well mixed.

Finally, we note that while some specific details of the mixing discussed here may be unique to the present geometry, it is expected that the principal findings of the present work - including the key mechanisms - will be applicable for a variety of initial conditions. In particular, the present results are representative of the general case in $\mathrm{R}-\mathrm{M}$ mixing in which the initial-condition perturbations are of larger scale, resulting in a post-shock flow that is dominated at early to intermediate times by a large-scale vorticity field operating on material interfaces established in the initial conditions.

In the future, planar velocity measurements could provide a valuable complement to the concentration measurements reported here. Alternative initial conditions might also be considered, such as a nominally one-dimensional air- $\mathrm{SF}_{6}$-air gas layer that may be more amenable to modelling, and/or extension to higher Mach numbers.

The authors would like to acknowledge S. Kearney for assistance with PLIF; B.J. Balakumar, A. Boyts, C. Goodenough, M. Marr-Lyon, and P. Vorobieff for experimental assistance; G. Weirs, V. Dwarkadas, and T. Plewa at the ASCI Flash Center at the University of Chicago for simulations of the initial conditions; B. J. Balakumar and D. Ranjan for reviewing the manuscript; fruitful discussions with R. Ristorcelli and E. Vold; and special thanks to R. Benjamin for significant contributions to experimental study of shock-accelerated flows and for pioneering our project.

\section{REFERENCES}

Adrian, R. J. 1991 Particle-imaging techniques for experimental fluid mechanics. Annu. Rev. Fluid Mech. 23, 261.

Arnett, W., Bahcall, J., Kirshner, R. \& Woosley, S. 1989 Supernova 1987a. Annu. Rev. Astron. Astrophys. 27, 629.

Brouillette, M. 2002 The Richtmyer-Meshkov instability. Annu. Rev. Fluid Mech. 34, 445.

Buch, K. \& DAHM, W. 1996 Experimental study of the fine-scale structure of conserved scalar mixing in turbulent shear flows. Part 1. Sc 》1. J. Fluid Mech. 317, 21.

Buch, K. \& DAнM, W. 1998 Experimental study of the fine-scale structure of conserved scalar mixing in turbulent shear flows. Part 2. $S c \approx 1$. J. Fluid Mech. 364, 1.

Dimotakis, P. 2000 The mixing transition in turbulent flows. J. Fluid Mech. 409, 69.

Eswaran, V. \& Pope, S. 1988 Direct numerical simulation of the turbulent mixing of a passive scalar. Phys. Fluids 31, 506.

GirimajI, S. 1992 On the modeling of scalar diffusion in isotropic turbulence. Phys. Fluids A 4, 2529.

HaAs, J. F. \& Sturtevant, B. 1987 Interaction of weak shock waves with cylindrical and spherical gas inhomogeneities. J. Fluid Mech. 181, 41.

JACOBS, J. 1992 Shock-induced mixing of a light-gas cylinder. J. Fluid Mech. 234, 629.

JACOBS, J. 1993 The dynamics of shock-accelerated light and heavy gas cylinders. Phys. Fluids A 5, 2239.

Kumar, S., Orlicz, G., Tomkins, C., Goodenough, C., Prestridge, K., Vorobieff, P. \& Benjamin, R. 2005 Stretching of material lines in shock-accelerated gaseous flows. Phys. Fluids 17, 1. 
Lindl, J., McCrory, R. \& CAMpbell, E. 1992 Progress toward ignition and burn propagation in inertial confinement fusion. Phys. Today 45, 32.

Lozano, A., Yip, B. \& Hanson, R. 1992 Acetone: a tracer for concentration measurements in gaseous flows by planar laser-induced fluorescence. Exps. Fluids 13, 369.

Marble, F. E. 1985 Growth of a diffusion flame in the field of a vortex. In Recent Advances in the Aerospace Sciences (ed. C. Casci), p. 395. Plenum.

Meshrov, E. 1969 Instability of the interface of two gases accelerated by a shock wave. Izv. Akad. Nauk. SSSR Mekh. Zhidk. Gaza. 4, 151.

Peng, G., Zabusky, N. \& Zhang, S. 2003 Vortex-accelerated secondary baroclinic vorticity deposition and late-intermediate time dynamics of a two-dimensional richtmyer-meshkov interface. Phys. Fluids 15, 3730.

PiCONE, J. M. \& BoRis, J. P. 1988 Vorticity generation by shock propagation through bubbles in a gas. J. Fluid Mech. 189, 23.

Prestridge, K., Vorobieff, P., Rightley, P. \& Benjamin, R. 2000 Validation of an instability growth model using particle image velocimetry measurements. Phys. Rev. Lett. 19, 4353.

QUiRK, J. J. \& KARNI, S. 1996 On the dynamics of a shock-bubble interaction. J. Fluid Mech. 318, 129.

Richtmyer, R. D. 1960 Taylor instability in shock acceleration of compressible fluids. Commun. Pure Appl. Maths 8, 297.

Rightley, P., VorobiefF, P., Martin, R. \& Benjamin, R. 1999 Experimental observations of the mixing transition in a shock-accelerated gas curtain. Phys. Fluids 11, 186.

Samtaney, R. \& Zabusky, N. 1994 Circulation deposition on shock-accelerated planar and curved density-stratified interfaces: models and scaling laws. J. Fluid Mech. 269, 45.

Su, L. \& CLEmens, N. 2003 The structure of fine-scale scalar mixing in gas-phase planar turbulent jets. J. Fluid Mech. 488, 1.

TAYLOR, G. I. 1950 The instability of liquid surfaces when accelerated in a direction perpendicular to their planes. Proc. R. Soc. Lond. A 201, 192.

Tomkins, C., Prestridge, K., Rightley, P., Marr-Lyon, M., Vorobieff, P. \& Benjamin, R. 2003 A quantitative study of the interaction of two Richtmyer-Meshkov-unstable gas cylinders. Phys. Fluids 15, 986.

Tomkins, C., Prestridge, K., Rightley, P., Vorobieff P. \& Benjamin, R. 2002 Flow morphologies of two shock-accelerated, unstable gas cylinders. J. Vis. 5, 273.

Weirs, G., Dwarkadas, V. \& Plewa, T. 2004 Flash validation for vortex-dominated flows. Tech. Rep. FLASH center at Univ. of Chicago.

YAng, J., Kubota, T. \& Zukowski, E. 1993 Applications of shock-induced mixing to supersonic combustion. AIAA J. 31, 854.

ZABUSKY, N. 1999 Vortex paradigm for accelerated inhomogeneous flows: visiometrics for the Rayleigh-Taylor and Richtmyer-Meshkov environments. Annu. Rev. Fluid Mech. 31, 495.

Zhang, S., Zabusky, N., Peng, G. \& Gupta, S. 2004 Shock gaseous cylinder interactions: dynamically validated initial conditions provide excellent agreement between experiments and numerical simulations to late-intermediate time. Phys. Fluids 16, 1203. 